\title{
Studies on Chitin VIIII. Some Properties of Water Soluble Chitin Derivatives
}

\author{
Seiichi TOKURA, Norio NishI, Akihiro TsUTSUMI, \\ and Oyin SOMORIN* \\ Department of Polymer Science, Faculty of Science, Hokkaido University, \\ Nishi 8-chome, Kita 10-jo, Kita-ku, Sapporo 060, Japan \\ *Chemistry Department, University of Lagos, \\ Akoka, Yaba, Lagos, Nigeria
}

(Received February 22, 1983)

\begin{abstract}
KEY WORDS Carboxymethyl-Chitin / Dihydroxypropyl-Chitin / IR Spectra / Polyelectrolyte / ${ }^{13} \mathrm{C}$ NMR / Reduced Viscosity / Intrinsic Viscosity / Alkali Chitin /
\end{abstract}

Chitin, one of the natural abundant polysaccharides present in the cuticles of Crustacea, is known to be insoluble in most common solvents except for strong acids such as methanesulfonic, sulfuric and formic acids. Therefore, litte is known about solution properties of chitin and its derivatives. The insolubility of chitin has been suggested to be due to its rigid crystalline structure through intra- and intermolecular hydrogen bonds. ${ }^{1}$ Attempts at disrupting these bonds in order to prepare soluble chitin derivatives have consisted in chemical modifications such as acylation ${ }^{2-5}$ or alkylation. ${ }^{6}$ However, the introduction of acyl or alkyl groups was found to enhance the solubility properties only in organic solvents ${ }^{2-5}$ and not in water. Ethylene glycol chitin is known as a water soluble chitin derivative and a substrate for lysozyme (EC 3.2.1.17). ${ }^{7,8}$ Carboxymethyl-chitin (CM-chitin), another water soluble chitin derivative, has previously been prepared by procedures cumbersome and time consuming. ${ }^{9,10} \mathrm{CM}$-chitin is a polyelectrolyte with properties resembling those of carboxymethyl-cellulose (CMC).

In the present study, CM-chitin and dihydroxypropyl-chitin (DHP-chitin) were prepared successfully by simple procedures involving freezing and the addition of a detergent such as sodium dodecylsulfate (SDS). The solution properties of the water soluble chitin derivatives were studied first. ${ }^{13} \mathrm{C}$ NMR spectroscopy was employed to investigate the initial alkylation sites on the $N$-acetylglucosamine residue in chitin molecules.

\section{EXPERIMENTAL}

\section{Materials}

Chitin was prepared from Queen Crab shells according to the method of Hackman ${ }^{11}$ and powdered to 45-60 mesh before use. Monochloroacetic acid, glycerol $\alpha$-monochlorohydrin and other reagents of reagent grade were obtained from Wako Pure Chemical Industries Ltd. and used without further purification.

\section{Preparation of Alkali-Chitin}

$10 \mathrm{~g}$ of chitin powder was suspended in $40 \mathrm{ml}$ of a freshly prepared $60 \%$ sodium hydroxide solution including $0.2 \%$ SDS at $4{ }^{\circ} \mathrm{C}$ and the slurry was kept in a freezer at $-20^{\circ} \mathrm{C}$ overnight after standing for $1 \mathrm{~h}$ at $4^{\circ} \mathrm{C}$. The alkali-chitin was used for alkylation reactions without further treatment so as to avoid deacetylation.

\section{Preparation of CM-Chitin}

The frozen alkali-chitin was suspended in $200 \mathrm{ml}$ of isopropyl alcohol at room temperature, and monochloroacetic acid was added in portions with mechanical stirring until the reaction mixture was neutralized. The product was filtered and washed with ethanol. The residue was extracted with 2 liters 


\section{S. Tokura et al.}

of water at room temperature with mechanical stirring. The water extract was slowly added to 5 liters of acetone to precipitate the CM-chitin. The precipitate was collected by centrifugation and washed with acetone several times. The CM-chitin $\mathrm{Na}$ salt was redissolved in water and the $\mathrm{pH}$ adjusted 2.0 by the addition of $2 \mathrm{~N} \mathrm{HCl}$ to give salt free CM-chitin. The solution was lyophilized after thorough dialysis against deionized water to remove any trace of salt. Yield, $8.9 \mathrm{~g}$.

\section{Preparation of DHP-Chitin}

DHP-Chitin was prepared by the reaction of alkali-chitin $(10 \mathrm{~g})$ with glycerol $\alpha$-monochlorohydrin (10 equivalent mol per mol of $\mathrm{N}$-acetylglucosamine residue) in isopropyl alcohol under conditions similar to those for the CM-chitin preparation. DHP-Chitin obtained was lyophilized after thorough dialysis against water. Yield, $10.3 \mathrm{~g}$.

\section{Estimation of the Degree of Substitution}

The degree of substitution was estimated by elemental analysis using Yanagimoto CHN Corder MT-2 and also by a potentiometric titration using Radiometer's Automatic Titor TTT1c-SBR2c under a nitrogen atmosphere in a $0.1 \mathrm{M} \mathrm{NaCl}$ solution at room temperature.

\section{${ }^{13}$ C NMR Measurement}

${ }^{13} \mathrm{C}$ NMR measurements were carried out with a JEOL FX-60Q $(60 \mathrm{MHz})$ spectrometer in $\mathrm{D}_{2} \mathrm{O}$ at $45^{\circ} \mathrm{C}$. The chemical shift due to the modified carbon at $\mathrm{C}_{6}$ or $\mathrm{C}_{3}$ position of the $\mathrm{N}$-acetylglucosamine residue as a result of alkylation was estimated. ${ }^{6}$

\section{IR Measurement}

IR spectra of chitin and chitin derivatives were recorded as $\mathrm{KBr}$ pellets on a JASCO infrared spectrophotometer A-302.

\section{Viscosity Measurement}

Viscosity of the solution of chitin derivatives was measured with an Ubbelohde type viscometer (flow time for water; $225 \mathrm{~s}$ ) at $25^{\circ} \mathrm{C}$. The ionic strength was adjusted with $\mathrm{NaCl}$. An aqueous solution of CM-chitin or DHP-chitin was dialyzed against deionized water or a sodium chloride solution of known concentration at room temperature. The concentration of chitin derivatives in the solution was estimated by micro-Kjeldahl nitrogen analysis. The intrinsic viscosity was obtained by plotting the reduced viscosity against the concentration of the chitin derivatives.

\section{RESULTS AND DISCUSSION}

High alkali concentration, the addition of SDS to facilitate the penetration of the alkali into chitin micelles and freezing for at least about $10 \mathrm{~h}$ were found adequate for preparing alkali-chitin. A quicker alternative procedure of freezing in a dry ice-acetone mixture or liquid nitrogen for a shorter period was not effective in preparing alkalichitin in high yield. The procedure employed for the preparation of chitin viscose $\mathrm{e}^{12}$ was also found unsuitable in this case because of the resulting higher degree of deacetylation. A lower alkaline concentration $(40 \%)$ resulted in a reduction of the yield of the water-soluble derivative. The water solubility of CM-chitin becomes apparent when the degree of susbstitution is over 0.6 .

DHP-chitin could be prepared under conditions similar to those for the CM-chitin. But the reaction proceeded under a strongly basic condition throughout the alkylation reaction, since chloride ion was not released as quickly from glycerol $\alpha$-monochlorohydrin as monochloroacetic acid. The solubility of DHP-chitin in water was difficult to maintain on storage over a long period of time. The solubility was found to be reduced markedly on storage for

Table I. Elemental analyses of CM- and DHP-chitins

\begin{tabular}{|c|c|c|c|c|c|c|c|}
\hline \multirow{2}{*}{ Sample } & \multicolumn{3}{|c|}{ Found $/ \%$} & \multicolumn{3}{|c|}{ Theoretical $/ \%$} & \multirow{2}{*}{$\mathrm{DS}^{\mathrm{a}}$} \\
\hline & $\mathrm{C}$ & $\mathrm{H}$ & $\mathrm{N}$ & $\mathrm{C}$ & $\mathrm{H}$ & $\mathrm{N}$ & \\
\hline CM-Chitin & 44.32 & 5.89 & 5.75 & 44.92 & 5.78 & 5.70 & 0.6 \\
\hline DHP-Chitin & 45.81 & 6.88 & 4.90 & 46.25 & 6.63 & 5.04 & 0.9 \\
\hline
\end{tabular}

${ }^{a}$ Degree of substitution was calculated on the basis that $1 / 2 \mathrm{H}_{2} \mathrm{O}$ was included per $N$-acetylglucosamine residue. 


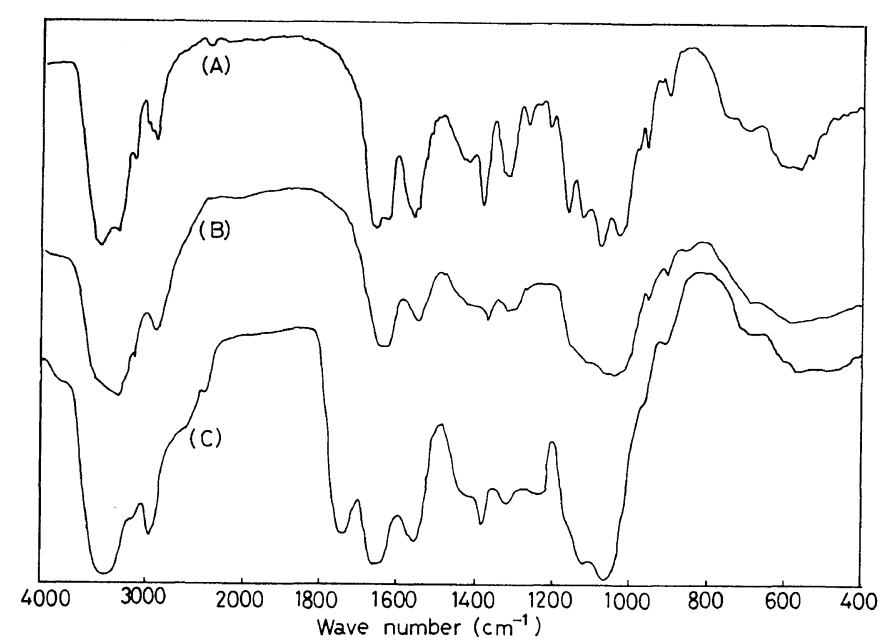

Figure 1. Infrared absorption spectra of (A) chitin, (B) dihydroxypropyl-chitin, and (C) carboxymethylchitin.

2-3 weeks at room temperature, but it was possible to redissolve the DHP-chitin in water by pretreatment with $6 \mathrm{M}$ urea at room temperature for $12 \mathrm{~h}$. This insolubility suggests the reformation of interand intramolecular hydrogen bonds between amide groups at the $\mathrm{C}_{2}$ position and hydroxyl groups of the glycerol residue. The degree of dihydroxypropylation was estimated to be 0.9 from elemental analysis, as shown in Table I.

The degree of carboxymethylation was estimated as $0.6-0.8$ under our reaction conditions from elemental analysis and potentiometric titration as shown in Table I. The $\mathrm{p} K_{\mathrm{a}}$ of the attached carboxyl group was found to be 3.40 from a potentiometric titration in $0.1 \mathrm{M} \mathrm{NaCl}$ and the content of the amino groups having a $\mathrm{p} K_{\mathrm{a}}$ of 6.40 was estimated to be less than $6 \%$. The amino group content was found to be $10 \%$ for DHP-chitin, a reasonable degree of deacetylation under our reaction conditions.

The IR spectra of chitin derivatives are shown in Figure 1 along with that of chitin. The absorption due to carbonyl stretching appears at $1730 \mathrm{~cm}^{-1}$ and that due to primary hydroxyl groups at 1070 $\mathrm{cm}^{-1}$ decreases on carboxymethylation. On the other hand, there is little difference between the IR spectra of DHP-chitin and chitin, since the addition of functional groups by this dihydroxypropylation amounts to only one secondary hydroxyl group per $\mathrm{N}$-acetylglucosamine residue.
The ${ }^{13} \mathrm{C}$ NMR spectra of chitin derivatives in $\mathrm{D}_{2} \mathrm{O}$ are shown in Figure 2. It is clearly evident that the peak intensity due to the $\mathrm{C}_{6}$ of $\mathrm{N}$-acetylglucosamine $(63.0 \mathrm{ppm})$ is reduced and a new peak can be observed at $69.0 \mathrm{ppm}$ due to the substituted carbon atom. There is no significant indication of a chemical shift due to the substituted $\mathrm{C}_{3}$ at $76.8 \mathrm{ppm}^{6}$ Consequently, the initial carboxymethylation site on the $\mathrm{N}$-acetylglucosamine was assumed to be the $\mathrm{C}_{6}$ position from the ${ }^{13} \mathrm{C}$ NMR spectrum. However, both hydroxyl groups at $\mathrm{C}_{6}$ and $\mathrm{C}_{3}$ were apparently substituted on dihydroxypropylation.

The CM-chitin behaved as a characteristic polyelectrolyte in aqueous solution as shown in Figure 3 . The reduced viscosity curve showed a maximum when the sodium chloride concentration was quite low. The reduced viscosity became proportional to the concentration of CM-chitin at high sodium chloride concentrations. The reduced viscosity values at various $\mathrm{CM}$-chitin concentration were almost identical with those of DHP-chitin, as shown in Figure 3(f) when the sodium chloride concentration was higher than $0.1 \mathrm{M}$. The intrinsic viscosity of $\mathrm{CM}$-chitin was calculated to be 5.2 in a $0.1 \mathrm{M} \mathrm{NaCl}$ solution at $25^{\circ} \mathrm{C}$ from the intercept of the reduced viscosity-concentration plot. The number average molecular weight of CM-chitin was thus estimated to be $1.63 \times 10^{5}$ by applying the viscosity equation proposed by Kaneko et al. ${ }^{13}$ in the os- 


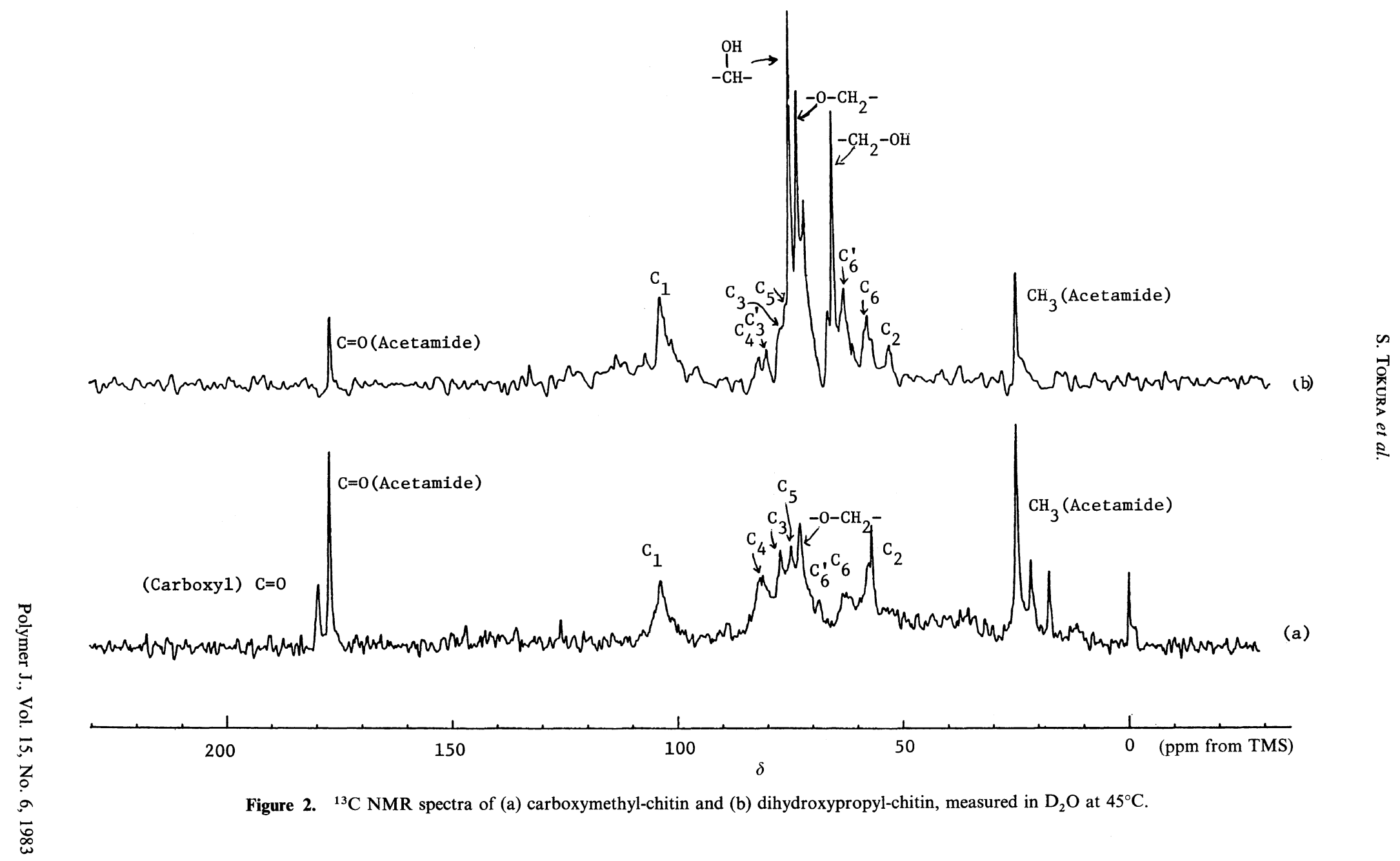




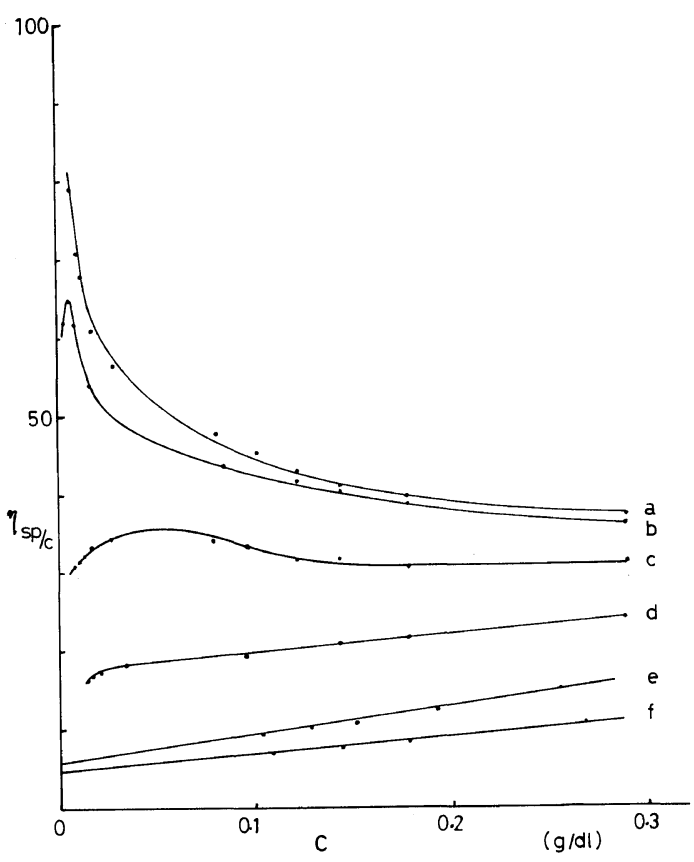

Figure 3. Relationship of reduced viscosity-concentration of chitin derivatives at $25^{\circ} \mathrm{C}$. $\mathrm{NaCl}$ concentrations are a, $0 ; \mathrm{b}, 0.0002 \mathrm{M} ; \mathrm{c}, 0.0008 \mathrm{M} ; 0.004 \mathrm{M}$; e, $0.05 \mathrm{M}$; f, 0.10 M. a-e, for CM-chitin; f, for DHP- and CM-chitins.

motic study of CM-chitin, $[\eta]=K M^{\alpha}$ where $K$ is $7.92 \times 10^{-5}$ and $\alpha$ is 1.00 . The estimated molecular weight of CM-chitin is slightly lower than that of chitin calculated by Hackman and Goldberg, ${ }^{14}$ but it is well known that the molecular properties of chitin depend on the source from which it came. The solution properties of $\mathrm{CM}$-chitin are now being investigated and compared with DHP-chitin.

Acknowledgement. The authors are very much indebted to Professor M. Kaneko of our department for his valuable comments in regard to the viscometric studies.

\section{REFERENCES}

1. F. G. Pearson, R. H. Marchessault, and C. Y. Liang, J. Polym. Sci., 43, 101 (1960).

2. N. Nishi, J. Noguchi, S. Tokura, and H. Shiota, Polym. J., 11, 27 (1979).

3. O. Somorin, N. Nishi, S. Tokura, and J. Noguchi, Polym. J., 11, 391 (1979).

4. K. Kaifu, N. Nishi, T. Komai, S. Tokura, and O. Somorin, Polym. J., 13, 241 (1981).

5. N. Nishi, H. Ohnuma, S. Nishimura, O. Somorin, and S. Tokura, Polym. J., 14, 919 (1982).

6. S. Tokura, J. Yoshida, N. Nishi, and T. Hiraoki, Polym. J., 14, 527 (1982).

7. K. Hamaguchi and M. Funatsu, J. Biochem., 46, 1059 (1959).

8. R. Senju and S. Okimasu, J. Agric. Chem. Soc. Jpn., 23, 432 (1950).

9. S. Okimasu, J. Agric. Chem. Soc. Jpn., 32, 471 (1958).

10. R. Trujillo, Carbohydr. Res., 7, 483 (1968).

11. R. H. Hackman, Austr. J. Biol. Sci., 7, 168 (1954).

12. J. Noguchi, O. Wada, H. Seo, S. Tokura, and N. Nishi, Kobunshi Kagaku, 30, 338 (1973).

13. M. Kaneko, Y. Inoue, and S. Tokura, Rep. Prog. Polym. Phys. Jpn., XXV, 759 (1982).

14. R. H. Hackman and M. Goldberg, Carbohydr. Res., 38, 35 (1974). 